\title{
Expectations of adolescents to receive reproductive health information and services from health service system: A qualitative study in Bangladesh
}

\author{
Samiha Yunus ${ }^{1}$, Sabrina Sharmin ${ }^{2}$, Nafisa Lira Huq ${ }^{3}$, Fariha Haseen ${ }^{4}$, Md Ali Imam ${ }^{5}$, Quamrun Nahar ${ }^{6}$ \\ ${ }^{1}$ Project Manager; ${ }^{4}$ Assistant Professor; Department of Public Health and Informatics (DPHI), Bangabandhu Sheikh Mujib Medical \\ University (BSMMU), Shahabag, Dhaka, Bangladesh. ${ }^{2}$ Independent Scholar, Mirpur, Dhaka, Bangladesh. ${ }^{3,6}$ Assistant Scientist; \\ ${ }^{5}$ Research Investigator; International Centre for Diarrheal Disease Research, Bangladesh (ICDDR,B), Mohakhali, Dhaka, Bangladesh.
}

\begin{abstract}
Adolescents, aged 10-19 years, constitute one fifth of Bangladesh's total population of 158 million. Similar to adolescents elsewhere, Bangladeshi adolescents also have special sexual and reproductive health (SRH) information and service needs; however, these needs are not adequately addressed by the existing health service system. The present study was conducted to identify adolescents' expectations of and preferences for receiving SRH information and services. This qualitative study was conducted in six purposively selected areas of Bangladesh using $20 \mathrm{Key}$ Informant Interviews (KII) during September-November 2012. Key informants aged between 15 to 19 years were leaders of youth organizations or class monitors in school. Informants were unmarried and married, males and females, and from rural and urban areas. This study identified that there is a strong need for SRH services for adolescents. Irrespective of their residence and gender, adolescents preferred health service providers to be qualified medical doctors, who are experienced, well-trained and polite. Availability of doctors, maintaining privacy and treatment through counseling were mentioned as their priorities. Both urban and rural adolescents recommended establishing adolescent friendly environment in every government and NGO health service facility. They also asked for gender specific service provider for female and male adolescents. Urban adolescents expressed the need for school health program with provision of school health clinics. On the other hand, rural adolescents recommended for community health workers to raise awareness on adolescent sexual and reproductive health (ASRH) issues with parents and families. The adolescents also expected to receive SRH information through mass media, school curriculum, and booklets on adolescent SRH, and peer educator. Addressing the expectations of adolescents will open a new skylight to policy makers to design highly accessible health services for adolescents in Bangladesh.
\end{abstract}

Keywords: Adolescent, Expectation, Sexual and reproductive health, Health information, Bangladesh.

\section{Introduction}

Adolescence (10-19 years) is a time in life that harbors many risks and dangers, but also one that presents great opportunities for sustained health and wellbeing through education and preventive efforts. Today, there are 1.2 billion adolescents in the world, forming 18 per cent of world population. ${ }^{1}$ The vast majority of them ( 88 per cent) live in developing countries. ${ }^{1}$ More than half the world's adolescents live in the South Asia, the East Asia and Pacific region, each of which contains roughly 330 million adolescents. ${ }^{1}$ There are 27.7 million adolescents in Bangladesh, making up about one fifth of the total population .

Today adolescents are growing up in circumstances quite different from their parents, with greater exposure to new ideas through media, internet, telecommunications and other avenues. ${ }^{3}$ Furthermore, the continuing growth in the absolute numbers of young people as well as the lengthening period of years spent unmarried (and in some cases sexually active) ensure a rapid and continuing growth in young people's need for sexual and reproductive health education. It should be underscored that the major health problems of young people are largely preventable. Research shows that adolescents have a high level of demand for accurate information. ${ }^{5}$

\section{Practice Points}

- Adolescents desired gender friendly one-stop service center in all tiers of government health settings for SRH information and services as well counseling.

- School-going adolescents thought of rescheduling the service day in line with their suitability e.g. during weekends

- Maintenance of confidentiality is the main concern for adolescents of all settings specially the urban school-going respondents assumed school health clinics could be a good option for maintaining their privacy.

- As per urban adolescents SRH information needs to be included in the regular school and college curriculum.

- Need awareness program for guardians and school teachers as well training on adolescent friendly health services for health providers.

Correspondence: Dr. Samiha Yunus, Department of Public Health and Informatics (DPHI), Bangabandhu Sheikh Mujib Medical University (BSMMU), Shahabag, Dhaka, Bangladesh. E-mail: dr.samihayunus@yahoo.com. 
To address global ASRH needs, Adolescent Friendly Health Services (AFHS) have been recognized as an appropriate and effective strategy in the International Conference on Population Development (ICPD) in Cairo, Egypt, 1994. However, expectation of adolescents to receive SRH information and services is still poorly understood. ${ }^{6}$ Despite of all the efforts adolescents' access to SRH services still remains poor in many settings. ${ }^{5,7,8}$ Providing youth-friendly health services alone may not be sufficient to increase adolescent's use of services. ${ }^{9}$ There is also a lack of use of electronic media to generate community support and stimulate parent-adolescent communication, community education and mobilization. Schools are currently an underutilized source of SRH information in the Bangladesh, although there have been recent efforts to introduce a curriculum based ASRH education program throughout the country. Besides, study suggests that teachers were not a preferred source of information. ${ }^{10}$

The need for context-specific research to support effective implementation of youth friendly health services has been noted. ${ }^{8,11}$ In this perspective, International Center for Diarrheal Disease Research, Bangladesh (icddr,b), conducted a qualitative study entitled "Updating frequently asked questions (FAQ) databank on adolescent sexual and reproductive health (SRH) in Bangladesh" which identified frequently asked questions of adolescents on sexual and reproductive health in Bangladesh. As a part of that study we conducted key informant interview to explore key issues that adolescents expect to receive SRH information and services from health service system in Bangladesh.

\section{Health Service Delivery System of Bangladesh}

The Ministry of Health and Family Welfare (MOHFW) organizes different programs to deliver health services and information. The programs are in turn implemented by the Directorate General of Health Services (DGHS) and Directorate General of Family Planning (DGFP). ASRH services are delivered through its program of the operational plans namely "Maternal, Neonatal, Child and Adolescent Health (MNCAH)" and "Maternal, Child, Reproductive and Adolescent Health (MCRAH)" respectively under DGHS and DGFP. ${ }^{12}$ Below is a brief description of the health service delivery system.

Services and information are provided through facilities and community outreach workers. At the village level, there is Community Clinic (CC) for a catchment population of about 6,000. The Community Health Care Provider (CHCP) provides healthcare and information from the $\mathrm{CC}$ as well as at the doorstep level. The Health Assistant (HA) and Family Welfare Assistant (FWA) provide services on healthcare (e.g., immunization, information of child health care) and family planning (FP) (e.g., pill and condom delivery and FP counseling and maternity-care information) through home visits. FWAs are female and HAs can be male or female.

There is Health and Family Welfare Center (H\&FWC) from where reproductive health $(\mathrm{RH})$ and FP services are provided. The female Family Welfare Visitor (FWV) provides FP services and antenatal care and male or female Sub-Assistant Community Medical Officer (SACMO) provide general healthcare from the H\&FWC and satellite clinics. Some H\&FWCs have a
Medical Officer (MO) who is a physician. There are some NGO health-service delivery programs that have facilities comparable to those H\&FWC and $\mathrm{CC}$ and community-based health workers comparable to HA and FWA.

The Upazila Health \& Family Planning Officer (UH\&FPO) is the health manager at the upazila level. There is a Upazila Health Complex (UHC) in every upazila to provide health services. In this facility there are posts of Residential Medical Officer (RMO); Junior Consultants (of Medicine, Gynecology, Surgery, Pediatrics), Anesthesiologist, Medical Officer (MO), Nurse Supervisor, Senior Staff Nurse, and Midwife. There is also a Upazila Family Planning Officer (UFPO) to supervise the DGFP activities in the upazila.

The district hospital is a tertiary-level hospital located at the district headquarters serve the entire district catchment population. The civil surgeon (CS) is the district health manager, and in-charge of a district hospital. Some district hospitals have superintendents to look after the hospital management related activities. A district hospital provides in- and out-patient services and specialized care. ${ }^{13}$

The Deputy Director of Family Planning (DDFP) is the district family planning manager. Family planning unit give services through Maternal and Child Welfare Center $(\mathrm{MCWC})$ in the district level.

Currently ASRH services and information are poorly delivered in the government health and family planning centers. The National Reproductive Health Strategy prioritizes four service areas in reproductive health: safe motherhood, family planning, MR and care for post abortion complications and management of RTI/STD. Both the DGHS and DGFP implement reproductive health services through their programs on $\mathrm{MNCH}$, reproductive and sexual health including family planning. There is also a 'Adolescent Health Strategy' and national standards to maintain the quality of adolescent health services ${ }^{12}$ However, evidence suggests that AFHS does not robustly and noticeably exist in the government health facilities in Bangladesh and existing policies. On other hand government health programs are also not addressing adolescents' needs properly . 5 Some NGOs provide ASRH services and information through their facility- and community-based service delivery systems but that are very limited and obviously not enough to meet adolescents' need.

\section{Materials and methods}

\section{Study Setting}

A quick review of ASRH programs in Bangladesh was done in 2012. Bangladesh is located in South-East Asia, bordered on the west, north, and east by a 4,095 kilometers $(2,545 \mathrm{mi})$ land frontier with India and, in the southeast, by a short land and water frontier (193 kilometers (120 mi)) with Myanmar. On the south is a highly irregular deltaic coastline of about 580 kilometers (360 mi), fissured by many rivers and streams flowing into the Bay of Bengal. Total population of Bangladesh is $166,280,712$.

Researchers did inventory of twenty three organizations working with adolescents and review their programs. 
The collected information was later verified with the organization for accuracy and completeness. For data collection researchers purposively selected 6 sites (Chakaria, Lalmonirhat, Naogaon, Dhaka, Khulna, and Narail) based on this organizational inventory from all over the Bangladesh considering both urban and rural areas. Among six study sites three sites were selected from urban areas (Dhaka, Khulna, and Lalmonirhat) and, three study sites were selected from rural areas (Chakaria, Naogaon, and Narail).

Dhaka is the capital of Bangladesh and located in central Bangladesh. Dhaka is one of world's most populated cities, with a population of about 15 million people. Dhaka District has an area of 1,463.60 square kilometers. Chakaria is an Upazila of Cox's Bazar District in the Division of Chittagong, Bangladesh. Cox's bazar is about $391 \mathrm{~km}$ from Dhaka. Chakaria Upazila has an area of $643.46 \mathrm{~km}^{2}$. Lalmonirhat is situated at the north side border of Bangladesh. It is a part of the Rangpur Division. It is about $343 \mathrm{~km}$ of Dhaka. Lalmonirhat District has an area of $1241.46 \mathrm{~km}$. Khulna is located in the Khulna Division. It is about $180 \mathrm{~km}$ from Dhaka. Khulna has an area of $4394.45 \mathrm{~km}^{2}$. Narail district is in south -western Bangladesh. It is a part of Khulna Division. It is about $130 \mathrm{~km}$ of Dhaka. Narail District has an area of 990.23 square kilometers. Naogaon district is located in the northern part of Bangladesh and is under Rajshahi Division. It is about $247 \mathrm{~km}$ of Dhaka.

\section{Sample}

This qualitative study was conducted to understand the expectations of adolescents from health service system in Bangladesh. A total of 20 key informants (eight males and 12 females) were purposively selected for the study. The all participants were 15 to19 years of age. As key informant, researcher only selected who were involved in adolescent programs or members of youth clubs/ organizations or youth leaders or class monitors in school having more knowledge about the adolescent sexual and reproductive health. Among the participants, 8 male and 6 female participants were unmarried and the rest six females were married. Eleven were from urban and nine from rural area of Bangladesh.

\section{Data Collection}

For data collection we used KII. Data collection was conducted during September 2012 to November, 2012. From each of the selected sites study participants were contacted through field workers of selected NGOs working in the area. In some cases assistance was sought from local schools and local community leaders for identifying study participants and locations to conduct the interviews. Each participant gave consent for the interview. Consent was also taken for conducting and recording of the interview. Pretested guidelines were used for interviews.

\section{Data Analysis}

The responses were transcribed and translated into English by maintaining the context of the responses. The transcripts of the KII were reviewed and the saturation level of information was considered that supported to determine the number of KII. After transcription and compilation of major findings the category identification in line with different background characteristics e.g. sex, marital status, urbanrural residence, were done through matrix analysis.
Cross interview analysis for each question was followed to see the similarities and differences of the data provided by the informants. From the beginning we undertook thematic analysis to understand adolescents' expectations about SRH information and services from the health-service system. In this way, content analysis through theme coding was used to analyze the qualitative data. To present the complex view of receiving SRH information and services, some verbatim statements of the study participants were directly quoted from the transcripts.

\section{Ethics approval and consent to participate}

The study obtained the ethical approval from the Research Review Committee and Ethical Review Committee of ICDDR,B. Participation in the study was voluntary. Informed written consent was obtained from each study participant before interview and audio recording of the interview. Confidentiality of informant was strictly maintained at all levels of data collection, processing and analysis.

\section{Results}

We synthesized the qualitative findings obtained from the KIIs. Based on our synthesis, following is a brief description about the adolescents' understanding and expectations about ASRH services in Bangladesh. Figure 1 is helpful in describing the results. Adolescents expect that ASRH services are composed of two distinct componentsinformation and health products - which can be obtained from the health system; and information can be obtained from a system beyond health. The health system in turn has two components-(a) health settings, facilities, or clinics and (b) community-based healthcare providers. They emphasize ASRH information as an important component of ASRH services. The adolescents eloquently narrated the sources of information and health products, the nature and characteristics of health settings and providers, barriers to ASRH service utilization and challenges of the ASRH service system. They underscored that an enabling and supportive environment has to be created to establish an effective and efficient ASRH service delivery system.

\section{Expectations about health settings}

\section{At facilities:}

Respondents put across despondency since adequate information is not provided to adolescents in government health service centers such as community clinic (CC), union health and family welfare centre (UH\&FWC), upazila health complex (UHC), and district hospital (DH). They recommended for establishing one-stop service center in government health settings so that they can get SRH information, services and counseling from one setting.

"There should be special provisions for adolescents in government health service centers where they can solve all their SRH problems and get the answers of all queries regarding SRH. "Urban, Unmarried, Male

Irrespective of their residence female respondents recommended for separate health centers for boys and 


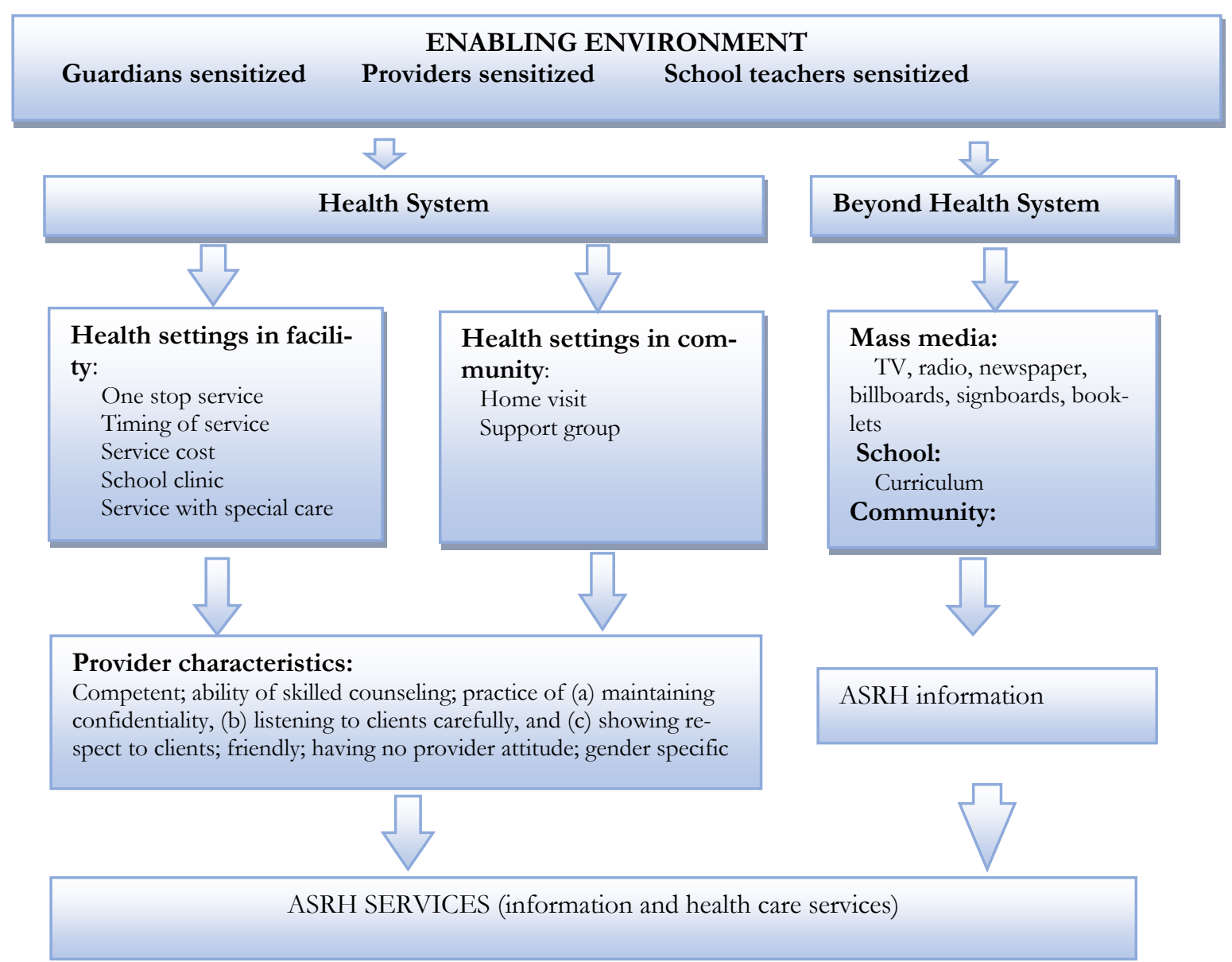

Figure 1: Expectations of study participants about ASRH services

girls. This is because girls do not want to encounter with boys while they are in the health facilities.

"There should be a separate health service centre for boys where they can talk freely, but in our government health service centre they cannot express themselves and no one wants to even listen them." Rural, Unmarried, Female

School-going respondents stated that time schedules of the health service centers are not suitable for the school going adolescents. They suggested fixing a day preferably in weekends so that adolescents can receive the SRH services. Some urban school-going respondents recommended for introducing school health clinics. They felt that service providers of school health clinic would be easily accessible and more privacy will be maintained.

In order to make adolescent SRH service delivery more effective rural respondents recommended engaging local government body in adolescent health service system. They thought that community clinics can also provide health information and services to rural adolescents.

"Government has to setup health service centers in the locality like a community clinic and has to arrange good doctors and health workers at those facilities with the help of the local chairman." Rural, Married, Female

Most of male respondents from rural areas preferred government facilities such as CC, UH\&FWC, and UHC. Male informants in rural areas urged for free treatment by introducing a special health card for adolescents through government health service centers.

"There should be a good doctor in government health service centre who will provide treatment to adolescents 1 or 2 days a week by using ID card and without payment." Rural, Unmarried, Male

Conversely most of the rural female respondents preferred services from NGO facilities in addition to government health services mainly because they felt that NGO facilities are adolescent friendly and provide quality services.

Irrespective of their residence all respondents suggested that health service centers should be more concerned about pregnant adolescents and should serve them with more care and responsibility.

"Special attention should pay to pregnant adolescent in the health service centre." Urban, Married, Female

At the community:

Rural respondents recommended involvement of community health workers, such as health assistant (HA), family welfare assistant (FWA), or NGO worker, in adolescent health service systems in addition to doctors. Irrespective of their residence females also recommended door to door service through home visit by community health workers. 
"In the villages, family planning officials regularly visit households, so they can also deliver the SRH information to adolescents. " Rural, Unmarried, Male

For receiving SRH information study participants also preferred involving community health workers to form adolescent support group in the community. The data indicated that the way of giving information can vary in rural and urban areas. For example they stated that urban adolescents have more access to internet and satellite channels so SRH information can be provided through these options. However in rural areas, there are community health workers (HA, FWA, and NGO worker) who have scope to work with rural adolescent. However, they strongly recommended providing similar SRH information all over the country including rural and urban areas.

\section{Expectations about health service providers}

Informants recommended that service providers should be qualified (having medical degree), experienced, well trained, having counseling skills, polite and should behave in a friendly manner with adolescents. Thus adolescents would feel encouraged to communicate freely with the service providers.

They also expected that service providers should greet adolescents sincerely at the health facilities. They expect that service providers should listen to their problems carefully and provide enough time. They also urged for friendly environment for adolescents in every government and NGO health service center. They identified medical doctors as a reliable source of SRH information and suggested appointing young doctors who will treat clients with dignity and respect without attitude.

"Adolescents will not feel free to share their sexual and reproductive health related problems with an old aged doctor." Urban, Unmarried, Female

The study participants identified that maintenance of confidentiality is main concern. Adolescents need assurance from service providers that their information would be kept confidential. Irrespective of respondents' residence and gender, respondents asked for female service providers for female adolescents and male service providers for male adolescents.

"They can easily open up and can share their problems with doctors if female doctors treat females and male doctors treat males. "Urban, Unmarried, Female

They recommended that doctors should be trained on adolescents SRH issues before providing services to them. The rural adolescent also urged for the special training of other service providers like health workers, nurses, and paramedics on counseling issue to develop the required skill to serve adolescents.

"Proper counseling should be provided to adolescents along with medical treatment and service providers should have special training on counseling." Urban, Unmarried, Female

\section{Expectations beyond health system}

Study adolescents preferred several means other than health service system to receive SRH information.
Mass Media:

Urban males and rural unmarried females recommended TV as a source of information. They suggested use of different programs, like dramas, for raising awareness. They also recommended radio programs, newspapers, billboards, and signboards through which ASRH information can be disseminated all over Bangladesh. However, urban respondents mentioned about cable channels for urban areas.

"TV channels or media can play a strong role for providing SRH information." Urban, Unmarried, Male

School-going adolescents from urban expressed their expectations by saying that SRH related booklets can be a source of information. They recommended providing SRH related booklets in the school library so that they can easily get those booklets and can learn by their own interest. Participants also suggested sending messages (SMS) on ASRH information through mobile phones.

School curriculum:

SRH information dissemination in schools and colleges was emphasized by school- going respondents from urban areas. According to them this information needs to be added to the school and college curriculum.

"Although we have a chapter of one page about AIDS, this is out of the syllabus. However, this type of information has to be added to our curriculum and syllabus. "Urban, Unmarried, Male

They recommended including the topic ASRH as a subject and subject marks should be counted in their grades. According to them, having this as part of an examination will make everyone take the subject seriously.

"If SRH related information is added to our curriculum, as a separate book and treated as a subject, and every school and college has a compulsory exam on it, then everyone will take this subject seriously." Rural, Unmarried, Male

Peer educators:

Half of the study participants strongly recommended establishing peer support groups. They considered that peers can convey all the information to others easily and effectively at the community level.

"Trained Peer can also use as source of information in the community. "Rural, Married, Female

Informants also identified that the educated adolescents can be a great resource. Through peer education it would be easy to educate those who cannot read message, out-of-school and married adolescents.

"Those who are educated can learn from schools, madrasa (Islamic education institute), or medical health care centers, or from any books. Then they can teach illiterate one by gathering them in a place." Rural, Married, Female

They also suggested GO-NGO collaboration to work with peer educator. 


\section{Expectations about enabling environment}

All the key informants commented that only provision of information to adolescents is not enough. Events or meetings can be arranged to sensitize guardians, service providers, and school teachers so that they realize the need for ASRH and subsequently support ASRH activities to enhance the ASRH service utilization. Study participants urged to engage school authorities (managing committee, governing body) to create enabling environment in schools.

"School authority and teacher should realize the importance of SRH education. Teachers should be trained on SRH issues so that they can discuss SRH issues without hesitation and clearly." Rural, Unmarried, Male

They maintained that guardians, community leaders, teachers, imam of the local mosque and other religious leaders have a vital role in every aspect of ASRH service delivery. Without changing the attitude of service provider, teachers and guardians it is unlikely to successfully introduce any health program for adolescents.

"Need to arrange awareness programs or training for guardians, community leaders and religious leaders, so that they cannot create any barrier." Urban, Unmarried, Male

\section{Discussion}

The various expectations of adolescents about SRH information and services highlight the need to strengthen the provision of high-quality information and services through multiple channels to reach in and out-of-school adolescents. These are "access" and "quality of care" issues. In the social context of Bangladesh, provision of comprehensive SRH services at a sole site can increase service utilization. The study adolescents expect adolescent friendly one-stop health service center with gender specific service provider. This finding is similar with other ASRH research findings in Africa. ${ }^{1}$ There are male and female service providers both at facilities and at the community and therefore the expectation of gender-specific provider can be met. The female respondents preferred NGO clinics; the main reasons are quality of care and maintenance of client privacy and confidentiality.

Rural male informants recommended for introducing free health services in the government health facilities for adolescents. Although the government health services are supposed to be free but in many cases service seekers have to procure the drugs from private sources. Research in other settings also found that free or affordable services, innovative financing mechanisms may increase demand and uptake of SRH services by adolescents. ${ }^{9}$ In Bangladesh, most of the adolescents do not have any income source of their own so investment is required in well-evaluated, context-specific strategies that aim to create a more supportive environment for adolescents.

Young people themselves, especially unmarried ones, are inhibited from using the present services mainly because of less friendly attitudes of providers as well as for their fear of disclosure of their privacy while they seek for care. For unmarried adolescents, there is an additional access issue resulting from provider attitude. In Bangladesh, for example, family planning services are said to be available only for eligible couples. Both facility- and community-level service providers not only hold relatively conservative attitudes to unmarried adolescent but also consider that the unmarried do not fall within the population that they expect to serve. ${ }^{10}$ Accordingly our study urban adolescents described the need for school health programs with provision of school health clinics to serve them. On other hand, rural adolescents described the need for sensitizing the service providers at the facilities (CC, UH\&FWC, UHC) and those work at the community (FWA, HA) for serving the adolescents with care and respect. This finding is very unique in this research. In Kenya and Zambia, provider training on attitudes, client confidentiality, communication, and counseling skills improved provider performance of serving adolescents more effectively. ${ }^{14}$ If government includes adolescent health competencies in basic pre-service training of all facility- and community-level providers it will be helpful to ensure adolescent friendly services in the country. In addition, the timing of government outdoor facilities overlaps with school timing. Respondents identified this issue as a barrier to access ASRH services. The study participants suggested taking initiative to sensitize school teachers for allowing adolescents to take time off for seeking health service during school hour.

Additionally, the study participants suggested that ASRH dissemination programs should be presented on a regular basis through newspapers, radio, and TV. TV program can be effective in raising awareness amongst married and adolescents, especially for those who cannot read messages. The participants also suggested arranging TV programs where adolescents could ask for information from a doctor over the phone. In this study adolescents identified a range of media, both private and public, as preferred sources of ASRH information, although media was not as prominent as has been reported in studies from Asia and Africa. ${ }^{15,16}$

According to current "Adolescent Health Strategy" in Bangladesh School Health Program and Bureau of Health Education of DGHS and Information, Education and Motivation Unit of DGFP is responsible for lifeskills education and correct information on physical and psychological changes. ${ }^{12}$ MOHFW, with TA support and in collaboration with Ministry of Education, takes initiative to formulate appropriate $\mathrm{RH}$ issues for inclusion in the school curricula. ${ }^{12}$ In our study school-going adolescents also expressed a preference for curriculumbased SRH information to be provided through schools. Evidence also suggests that skill-focused curriculumbased ASRH education improves knowledge, reduces risk behavior and, to some extent, improves SRH outcomes. ${ }^{10}$ The study participants recommend establishing peer support group who can educate those adolescents of any marital status who cannot read messages. Studies found that friends were a common source of SRH information; however, some other studies raised controversy since peer-group performance found to be less than satisfactory. ${ }^{17}$

Communication gap between parent and adolescent on $\mathrm{SRH}$ issues continued to be a socio-cultural barrier in Bangladesh. ${ }^{5}$ Adolescent SRH programs which covered culturally sensitive or controversial topics and gained 
approval from community influential persons and guardian effectively increased service utilization. ${ }^{10}$ The study informants noted that parents need to be sensitized and involved in designing ASRH interventions. This will help to create positive changes among the gatekeepers of the adolescents.

We would like to register a limitation of our qualitative study is that generalization of the findings may not be possible with the small number of respondents.

\section{Conclusion}

Despite of having robust health service provision in rural settings of Bangladesh from ward and union to upazila level, lack of separate space especially for adolescents seems to be the prior concern. Consequently, the rural adolescents expected to have adolescent friendly one-stop service centre. Furthermore, to ensure the enabling environment of the one stop service centre the health systems of Bangladesh should address the issues regarding responsiveness of the providers to adolescents e.g. availability of gender specific doctors; polite behavior; maintenance of privacy and confidentiality; time consuming counseling etc. In addition, considering privacy adolescent friendly environment at all service provisions was the prior expectation of the urban adolescents. Urban adolescents expressed the need for school health program through school health clinic and rural adolescents recommended for community health workers to raise awareness on adolescent sexual and reproductive health (ASRH) issues with parents and families. The adolescents also expected to receive SRH information through mass media, school curriculum, and booklets on adolescent SRH, and also through peer educator. Therefore, promotion of age appropriate comprehensive sexuality education, which are on par with international standards, is urgently needed through all academic and training institutions.

However, in Bangladesh adolescents' need of SRH information and services is not addressed so far according to their expectation. Moreover, adolescents' views should be urgently addressed to do advocacy for comprehensive policy and program development, investments and implementation in designing their adolescent targeted health services. Addressing the expectations of adolescents will open a new skylight to policy makers to design highly accessible health services for adolescents in Bangladesh as well to build capacity for the delivery of age and gender sensitive sexual and reproductive health services. Finally, this study findings indicates a way forward to create a robust system for data collection/analysis on the sexual and reproductive health of adolescents, including unmarried adolescents, to inform policy and programming.

\section{Competing interest}

All authors declare that they have no competing interests.

\section{Acknowledgement}

This study was funded by University Research Co., LLC (URC). ICDDR,B acknowledges with gratitude the commitment of URC to its research efforts. icddr, $b$ is also grateful to the Governments of Bangladesh, Canada, Sweden and the UK for providing core/unrestricted support.
The study is also thankful to all adolescents, technical interest group (TIG) members, expert panel members and data collectors who contributed in this study. The authors gratefully acknowledge to Mizanur Rahman (PhD) Senior Research Advisor, MEASURE Evaluation, University of North Carolina, Chapel Hill. (www.cpc.unc.edu/measure) and Jocalyn Clark (MSc $\mathrm{PhD}$ ); Executive Editor \& Scientific Writing Specialist Communications \& Development; ICDDR,B, Dhaka, Bangladesh (www.icddrb.org) for their review.

\section{References}

1. United Nations Children's Fund (UNICEF). The State of the World's Children 2011. Adolescence: An Age of Opportunity. New York: UNICEF, 2011.

2. United Nations Children's Fund (UNICEF) Bangladesh. The children: Adolescence. http://www.unicef.org/bangladesh/ children 356.htm (accessed on September 2018)

3. Hindin MJ, Fatusi AO. Adolescent sexual and reproductive health in developing countries: an overview of trends and interventions. Int Perspect Sex Reprod Health. 2009; 35(2)58-62.

4. Shaw D. Access to sexual and reproductive health for young people: bridging the disconnect between rights and reality. Int $J$ Gynecol Obstet. 2009; 106(2):132-36.

5. Raphael D. Determinants of health of NorthAmerican adolescents: evolving definitions, recent findings, and proposed research agenda. $J$ Adolescent Health. 1996; 19(1):6-16.

6. Abajobir AA, Seme A. Reproductive health knowledge and services utilization among rural adolescents in east Gojjam zone, Ethiopia: a community-based cross-sectional study. BMC Health Serv Res. 2014; 14(1):138.

7. Guse K, Levine D, Martins S, Lira A, Gaarde J, Westmorland W, Gilliam M. Interventions using new digital media to improve adolescent sexual health: a systematic review. $J$ Adolescent Health. 2012; 51(6); 535-43.

8. World Health Organization. Adolescent friendly health services: an agenda for change. Geneva: WHO, 2002.

9. Kesterton AJ, Cabral de Mello M. Generating demand and community support for sexual and reproductive health services for young people: A review of the Literature and Programs. Reprod Health. 2010; 7:25.

10. Kennedy EC, Bulu S, Harris J, Humphreys D, Malverus J, Gray NJ. Be kind to young people so they feel at home": a qualitative study of adolescents' and service providers' perceptions of youth-friendly sexual and reproductive health services in Vanuatu. $B M C$ Health Serv Res. 2013; 13(1):455.

11. World Health Organization. Making health 
services adolescent friendly: Developing national quality standards for adolescent friendly health services. Geneva: WHO, 2002.

12. Ministry of Health and Family Welfare (MOH\&FW), Government of the People's Republic of Bangladesh. Health Population \& Nutrition Sector Strategic Plan (HPNSSP), 2011-2016. Dhaka: MOH\&FW, 2010.

13. Government of the People's Republic of Bangladesh, Ministry of Health and Family Welfare. Health Bulletin, 2014. Dhaka: Management Information System, Directorate General of Health Services, 2014.

14. Warenius LU, Faxelid EA, Chishimba PN, Musandu JO, Ong'any AA, Nissen EB. Nurse-midwives' attitudes towards adolescent sexual and reproductive health needs in Kenya and Zambia. Reprod Health Matters 2006; 14 (27):119-28.
15. Gold J, Pedrana AE, Sacks-Davis R, Hellard ME, Chang S, Howard S, Keogh L, Hocking JS, Stoove MA. A systematic examination of the use of online social networking sites for sexual health promotion. BMC Public Health. 2011; 11(1):583.

16. Hackett KM, Mukta US, Jalal CS, Sellen DW A qualitative study exploring perceived barriers to infant feeding and caregiving among adolescent girls and young women in rural Bangladesh. BMC Public Health. 2015; 15(1):771.

17. World Health Organization. A Framework for the Integration of Adolescent Health and Development Concepts Into Pre-service Health Professional Educational Curricula. Geneva: WHO, 2002. 